\title{
Imunosuppresive Activity of Momordica charantia L. fruit extract on the NF-kB pathway in Drosophila melanogaster
}

\author{
Nurfadhilah Asfa 1(D), Ulfah Mahfufah 1(D), Muhammad Khadafi Anugrah Pratama 1(D), Reski Amalia \\ Rosa $^{1(D)}$, Nur Rahma Rumata ${ }^{2}$ (D), Firzan Nainu 1,*(D)
}

1 Faculty of Pharmacy, Universitas Hasanuddin, Makassar 90245, Indonesia

2 Sekolah Tinggi Farmasi Makassar, Makassar 90242, Indonesia

* Correspondence: firzannainu@ unhas.ac.id (F.N.);

Scopus Author ID 57120069200

Received: 15.09.2021; Revised: 10.10.2021; Accepted: 14.10.2021; Published: 20.11.2021

\begin{abstract}
The activation of the NF-kB pathway leading to the production of proinflammatory cytokines is a critical feature in innate antiviral immunity. However, in SARS-CoV-2 infection, a high number of cases with a prolonged late-stage stimulation of cytokine production that leads to a cytokine storm phenotype, an undesirable, dangerous immune-related state that can cause multiple organ failures, have been reported. To treat this, immunosuppressants with selective action on the innate NF-kB pathway are urgently required. Bitter gourd (Momordica charantia L.) has been reported to yield antiinflammatory activity, thus might be a potential candidate for such effort. In this study, we carried out experimental procedures on the PGRP-LB mutant line of Drosophila melanogaster to examine the immunosuppressive effect of Momordica charantia L. fruit extract (MCFE) on the NF-kB pathway. Initial phytochemical screening revealed that Momordica charantia L. fruit extract contains alkaloids, flavonoids, tannins, and saponins. Furthermore, the phenotypical analysis demonstrated that MCFE could improve the survival and locomotor of the PGRP-LB mutant line of Drosophila melanogaster in a concentration-dependent manner. Additional gene expression analysis revealed that the expression of $d p t$ and dro, two important downstream genes in the Imd (NF-kB) pathway of D. melanogaster, was significantly reduced, in a different expression profile, in response to MCFE treatment. However, it is important to note that while the expression of $d p t$ was dramatically repressed in all extract-treated groups, the expression of dro occurred in a concentration-dependent manner. These results strongly support the notion that Momordica charantia L. can reduce the expression of proinflammatory cytokines downstream of the NF-kB pathway, hence potential to be used as a source candidate to harvest prospective immunosuppressive compounds to alleviate the cytokine storm condition.
\end{abstract}

Keywords: bitter gourd; Imd pathway; antimicrobial peptides; immunosuppressant.

(C) 2021 by the authors. This article is an open-access article distributed under the terms and conditions of the Creative Commons Attribution (CC BY) license (https://creativecommons.org/licenses/by/4.0/).

\section{Introduction}

COVID-19, an infectious disease caused by SARS-CoV-2, has spread throughout the world, causing a pandemic. A high number of COVID-19 cases, more than 200 million cases with almost 5 million deaths, has been reported as of September 14, 2021 [1]. COVID-19 is characterized by respiratory system disorders, similar to the coronavirus-related diseases reported before, SARS and MERS [2,3]. In the COVID-19 pandemic, the immune system will play an important role in maintaining the host condition. The activation of the innate and adaptive immune system upon introducing the SARS-CoV-2 into the body shall lay a beneficial status to the infected human hosts in this condition [4,5]. 
As an initial innate antiviral response to SARS-CoV-2 infection, proinflammatory cytokines are robustly produced. However, late-stage expression of proinflammatory cytokines during SARS-CoV-2 infection can provoke the induction of cytokine storm that leads to multiple organ failures [5,6]. Cytokine storms can increase the risk of death for COVID-19 patients. Thus immediate suppression of cytokine production is urgently required. In the case of cytokine storm, certain proinflammatory cytokines such as IL-2, IL-1, IL-6, IFN-MIP1 $\alpha$, $\mathrm{MCP} 1$, and TNF- $\alpha$ have been reported to cause inflammation, primarily in the respiratory system $[5,6]$.

Activation of NF- $\kappa B$ (Nuclear Factor Kappa Beta) via the myeloid differentiation primary response 88 (MyD88) pathway using pattern-recognition receptors (PPRs) is one of the canonical mechanisms for the induction of inflammation in patients with COVID-19 [7]. Thus, inhibition of the NF- $\kappa$ B pathway shall play a role in alleviating inflammation that may improve lung condition. This, in turn, shall increase the likelihood of host survival [8].

In vitro studies demonstrated that SARS-CoV-2 spike proteins strongly induce cytokine responses via the NF- $\mathrm{KB}$ pathway $[9,10]$. Currently available drugs, such as glucocorticoids, can increase the expression of I $\mathrm{B}$, which can inhibit NF- $\kappa \mathrm{B}$ in the cytoplasm, thereby reducing cytokine levels [6]. Corticosteroids have been suggested as one of the potential immunosuppressive drugs for treating COVID-19 patients suffering from cytokine storms $[6,8]$. In addition to that, the use of interleukin (IL)-6 inhibitors is a viable option in managing cytokine storm-related conditions $[11,12]$. However, these drugs have some disadvantages, such as being expensive (IL-6 inhibitors) and can cause unintended organ disorders (corticosteroids) [5,6,12]. Therefore, alternative immunosuppressive agents with potent pharmacological activity to alleviate cytokine overexpression, selective mechanism of action, and less adverse events are urgently required.

Previous studies have found that suppression of NF- $\kappa \mathrm{B}$ activation may be an alternative approach for treating patients with cytokine storms [6,8,13]. Bitter gourd (Momordica charantia L.) contains several compounds such as glycosides, saponins, alkaloids, triterpenoids, steroids, proteins, and specific compounds, namely momocharin, known to yield anti-inflammatory activity $[14,15]$. According to Chao et al. (2014), the secretion of proinflammatory cytokines such as IL-1, IL-6, and TNF- $\alpha$ decreased significantly with oral administration of Momordica charantia L. extract. It was thought to be due to decreased activity of $\mathrm{NF}-\mathrm{\kappa B}$, a transcription factor that plays a role in producing components of the immune and inflammatory system [16]. However, whether Momordica charantia L. fruit extract (MCFE) has any selective activity on the inhibition of NF- $\mathrm{BB}$ transcription factors and plays a pharmacological role in alleviating cytokine storm-related pathologies and improving the host's host survival remains to be determined.

Based on such notion, we further investigated the pharmacological effect of MCFE on the NF- $\mathrm{BB}$ pathway in a genetically amenable model system, Drosophila melanogaster. This insect has about $70 \%$ genetic similarity with humans, is inexpensive, easy to maintain, faster growth, and easy to manipulate genetically, all advantages that can facilitate robust and translatable research $[17,18]$. In addition to that, the use of D. melanogaster as an in vivo platform in this study provides several advantages for researchers in developing countries, including Indonesia. Moreover, D. melanogaster has been known to express Relish, a transcription factor homologous to the human NF- $\kappa$ B. The translocation of Relish into the nuclear compartment (Imd pathway) promotes the expression of several antimicrobial peptides (AMPs), proteins considered as an equivalent of the human cytokines [19]. The availability of 
mutant lines carrying an increased expression of AMPs, somewhat resembling the cytokine storm condition, is one of the reasons why D. melanogaster was chosen as a model organism in this particular study. The results obtained here shall provide a basic overview of the effect of MCFE on the NF- $\mathrm{KB}$ pathway and how such treatment can alleviate the overexpression of AMPs and resolve the inflammation.

\section{Materials and Methods}

\subsection{Fly stocks.}

This study used the fruit fly Drosophila melanogaster genotype Oregon R (wildtype) and PGRP-LB ${ }^{\Delta}$ (mutant line lacking PGRP-LB expression) as model organisms. The lack of PGRP-LB has been shown to induce the overactivation of NF- $\kappa B$ (Imd) pathway in $D$. melanogaster upon introducing a proper ligand [20]. Both fly lines were obtained from the Laboratory of Host Defense and Responses (Kanazawa University, Japan) and were steadily maintained in the culture vials under standard conditions (12 hours light and 12 hours dark cycles, $25^{\circ} \mathrm{C}$, and standard cornmeal-based food) based on our established procedure $[21,22]$. The age of Drosophila used in the experiment was 5-7 days.

\subsection{Sample preparation and extraction of Momordica charantia L. fruit.}

Momordica charantia L. fruit samples were obtained from the Gowa regency, South Sulawesi, Indonesia. Samples were washed in running water, weighed, sliced into small pieces, and dried in the oven at $60^{\circ} \mathrm{C}$. After drying, samples were weighed and processed into dried simplicia prior to the maceration procedure using $96 \%$ ethanol ( 24 hours for 3 days at room temperature). The obtained filtrate was collected and evaporated at a temperature of $55^{\circ} \mathrm{C}$ until a thick extract, namely Momordica charantia L. fruit extract (MCFE), was obtained.

\subsection{Phytochemical screening.}

Momordica charantia L. fruit extract (MCFE) was subsequently subjected to basic phytochemical screening tests to detect the presence of alkaloid, tannin, flavonoid, terpenoid steroid, and saponin, based on the established protocols [23]. Briefly, in the alkaloid test, MCFE was mixed with $2 \mathrm{~mL}$ of chloroform and $2 \mathrm{~mL}$ of ammonia and filtered. Approximately 3-5 drops of concentrated $\mathrm{H}_{2} \mathrm{SO}_{4}$ were added to the filtrate, and the mixture was shaken until the formation of two layers was observed. The upper layer was analyzed with 4-5 drops of Dragendorff's reagent. In the tannin test, MCFE was mixed with 10 drops of $10 \% \mathrm{FeCl}_{3}$ and observed for a blackish-green or blue-black color. MCFE was mixed with $100 \mathrm{~mL}$ of hot water in the flavonoid test, boiled for 5 minutes, then filtered. Approximately $0.05 \mathrm{~g}$ of $\mathrm{Mg}$ powder and $1 \mathrm{~mL}$ of concentrated $\mathrm{HCl}$ were mixed with $5 \mathrm{~mL}$ of the filtrate, and the mixture was subjected to vigorous shaking. A positive flavonoid test is indicated by the formation of a red, yellow, or orange color. In the terpenoid steroid test, MCFE was mixed with 10 drops of glacial $\mathrm{CH}_{3} \mathrm{COOH}$ and 2 drops of concentrated $\mathrm{H}_{2} \mathrm{SO}$. The resulting mixture was gently shaken and left for a few minutes prior to observation of color changes. Blue or green color indicates the presence of steroid compounds, while the formation of red or purple indicates the presence of triterpenoids. Lastly, in the saponin test, MCFE was mixed with $10 \mathrm{~mL}$ of water, shook for 1 minute, and mixed with 2 drops of $1 \mathrm{~N} \mathrm{HCl}$. The formation of foam that remains stable for more than 7 minutes indicates a positive result. 


\subsection{Survival assay.}

Two survival assays were carried out in this study. In the survival assay, each group consisted of ten female flies at the age of 5-7 days determined from the first day of emergence from their pupal case. The first survival assay was performed using Oregon R (wildtype $D$. melanogaster) and PGRP-LB ${ }^{\Delta}$ (mutant line lacking for PGRP-LB expression) to examine the effect of PGRP-LB gene knockout on the lifespan of the fruit fly. The second survival assay was carried out using only the PGRP-LB ${ }^{\Delta}$ line to investigate the effect of treatment on the survivorship changes in the respective fly line. All PGRP-LB ${ }^{\Delta}$ groups were monitored for their survival rates based on the following procedure. Briefly, females of D. melanogaster were separately assigned into groups. All flies were fed with standard food prior to survival assay testing. One group of flies was designated as the healthy control group (fed with standard fly food), and another one was assigned as the solvent control group (fed with ethanol 96\%containing fly food). Three groups, designated as treatment groups, were subjected to MCFE treatments at a concentration of $2 \%, 0.2 \%$, and $0.02 \%$. A group of flies treated with dexamethasone was used as a positive control group. All foods were changed every three days, and flies were maintained until the end of observation.

\subsection{Locomotor assay.}

All experimental groups were observed for locomotors based on the negative geotaxis method with some modifications [24]. Experimental flies were placed in an empty vial that had been marked. The vial is then tapped to ensure the fly is in the same starting position. Observations were made for 15 seconds after tapping. Flies that cross the line are counted.

\subsection{Gene expression analysis.}

Total RNA isolation was carried out in all fly groups based on our established protocols $[22,25,26]$, with slight modifications. Five live flies from each group were transferred to Treff tubes and then stored in a freezer at $-80^{\circ} \mathrm{C}$ prior to total RNA extraction using the Wizard SV Total RNA Isolation System (Promega ${ }^{\circledR}$ ). RNA concentration of each sample was measured using a nano-spectrophotometer (BioDrop ${ }^{\circledR}$ ) prior to subsequent amplification using reverse transcriptase quantitative PCR (RT-qPCR) method in RotorGene Q thermal cycler (Qiagen, Germany). The expressions of $d p t$ (Diptericin) and dro (Drosocin) were examined quantitatively by RT-qPCR method based on the use of a set of $d p t$ primers ( $d p t$ forward primer sequence: 5'-AGGTGTGGACCAGCGACAA -3' and dpt reverse primer sequence: 5'TGCTGTCCATATCCTCCATTCA-3') and dro primers (dro forward primer sequence: 5'TCCACCACTCCAAGCACAATG-3' and dro reverse primer sequence: 5'ACACATCTTTAGGCGGGCAG-3') in a reaction volume of $10 \mu$ l using the GoTaq ${ }^{\circledR} 1-$ Step RT-qPCR System (Promega ${ }^{\circledR}$ ) according to the manufacturer's instructions. A standard melt curve analysis was carried out to verify the presence of the expected PCR products. The expression level of ribosomal protein rp49, used as an internal RT-qPCR control, was assessed using a set of rp49 primers ( rp49 forward primer sequence: 5'AGATCGTGAAGAAGCGCACCAAG-3' and rp49 reverse primer sequence: 5'CACCAGGAACTTCTTGAATCCGG-3'). The following RT-qPCR running profiles were used: $37^{\circ} \mathrm{C}$ for 15 minutes (reverse transcription step), $95^{\circ} \mathrm{C}$ for 10 minutes (hot-start step), and a set of amplification profiles at $95^{\circ} \mathrm{C}$ for 10 seconds, $60^{\circ} \mathrm{C}$ for 30 seconds, and $72^{\circ} \mathrm{C}$ for 30 
seconds for 40 repeated cycles ended with melt curve analysis from $60^{\circ} \mathrm{C}$ to $95^{\circ} \mathrm{C}$. The data were analyzed using the relative quantification method.

\subsection{Data processing.}

Data obtained from the survival test was processed using the Kaplan-Meier approach and statistically analyzed using the LogRank method. Data obtained from the locomotor test and gene expression analysis were presented as mean \pm S.D. and analyzed using the One Way ANOVA method. All statistical analyses were carried out using GraphPad Prism ${ }^{\circledR} 8$.

\section{Results and Discussion}

\subsection{Phytochemical content of Momordica charantia L. fruit extract.}

The results of the phytochemical screening of MCFE are summarized in Table 1. Based on the table below, it is apparent that MCFE contains tannins, flavonoids, alkaloids, and saponins. Alkaloids have been known to yield anti-inflammatory activity, as evidenced by a decrease in the production of IL-1, IL-2, IL-6, and TNF- $\alpha$ [27]. In addition, several flavonoid compounds have been reported to exert antioxidant activity that can prevent inflammation and tissue damage [28].

Table 1. Results of phytochemical screening of Momordica charantia L. fruit extract.

\begin{tabular}{c|l|c|l} 
No & \multicolumn{1}{|c|}{ Test } & Results & \multicolumn{1}{c}{ description } \\
\hline 1 & Tannins & + & Change color to black \\
\hline 2 & Flavonoids & + & Color change to yellow \\
\hline 3 & Alkaloids & + & There is a white precipitate \\
\hline 4 & Steroids/Terpenoids & - & No change to blue/red \\
\hline 5 & Saponins & + & Foam lasts up to 7 minutes
\end{tabular}

3.2. The loss of PGRP-LB leads to a shorter fly lifespan, and this is possibly due to NF- $\kappa B$ overactivation.

PGRP-LB has been known as a negative regulator of NF- $\kappa B$ (Imd) activation [20]; thus, the loss of PGRP-LB plays a tremendous role in the overactivation of the NF- $\mathrm{BB}$ (Imd) pathway in the presence of a proper ligand. To test the effect of such phenotype on the survival of flies, a simple survival assay was carried out.

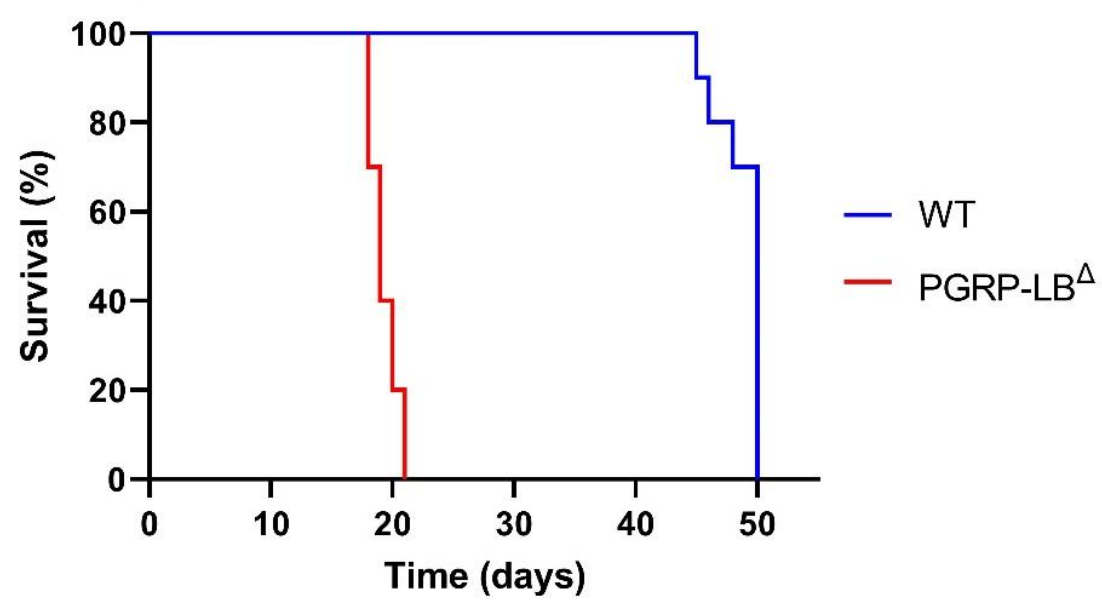

Figure 1. Lifespan of WT and PGRP-LB ${ }^{\Delta}$ D. melanogaster. Mutant line lacking for PGRP-LB experienced early death phenotype compared to the WT flies in presence on innocuous infection. WT, wildtype. 
As shown in Figure 1, PGRP-LB ${ }^{\Delta} D$. melanogaster experienced early death upon the loss of PGRP-LB, in the presence of innocuous infection, compared to its Oregon R wildtype counterparts. The survival of PGRP-LB ${ }^{\Delta}$ D. melanogaster is shorter due to the loss of PGRPLB protein, which functions to maintain the regulation of the NF-kB pathway at a homeostatic condition, in the presence of a proper ligand, for example, gut bacterial peptidoglycan. In the absence of PGRP-LB, the expressions of Diptericin and Drosocin, two NF- $\mathrm{BB}$ (Imd)-mediated antimicrobial peptides (AMPs), were upregulated (Figure 2), much likely as a result of overactivation NF- $\kappa \mathrm{B}$ (Imd). These results are in line with the ones reported by others [20].

As shown in Figure 2, the expression of $d p t$ and dro genes (encodes for Diptericin and Drosocin, respectively) in the PGRP-LB ${ }^{\Delta}$ flies was significantly increased compared to those observed in its wildtype counterpart. These genes are expressed through the Imd (immune deficiency) pathway, where this pathway is regulated by NF- $\kappa B$ (Relish). Overactivation of NF-kB has been suggested to be associated with neurodegeneration and a shorter lifespan [29].

A

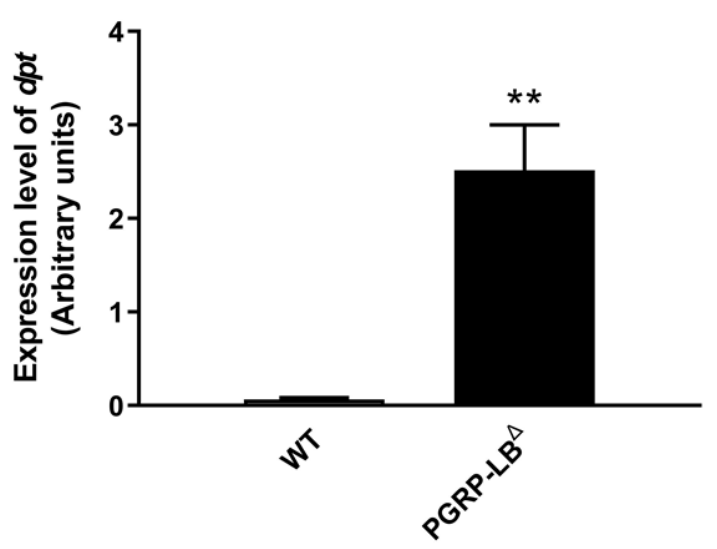

B

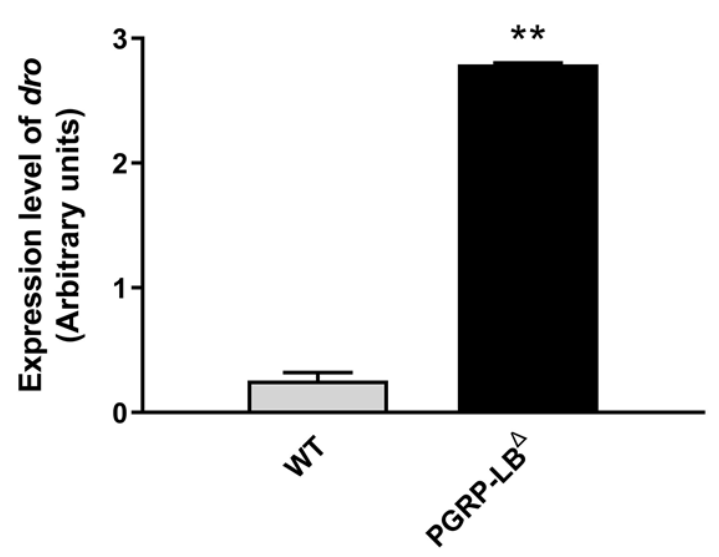

Figure 2. Results of gene expression analysis of $d p t(\mathrm{~A})$ and $d r o(\mathrm{~B})$ in Oregon $\mathrm{R}$ and PGRP-LB ${ }^{\triangle} D$. melanogaster. Adult fruit flies aged 5-8 days were subjected to intended groups. Flies fed with standard food and received no additional treatment. $* *, p<0.01$

\subsection{Momordica charantia L. fruit extract prolonged the survival and slightly enhanced the} locomotor of the PGRP-LB mutant flies.

The anti-inflammatory effect of MCFE was previously reported in the mammalian model system [14-16].

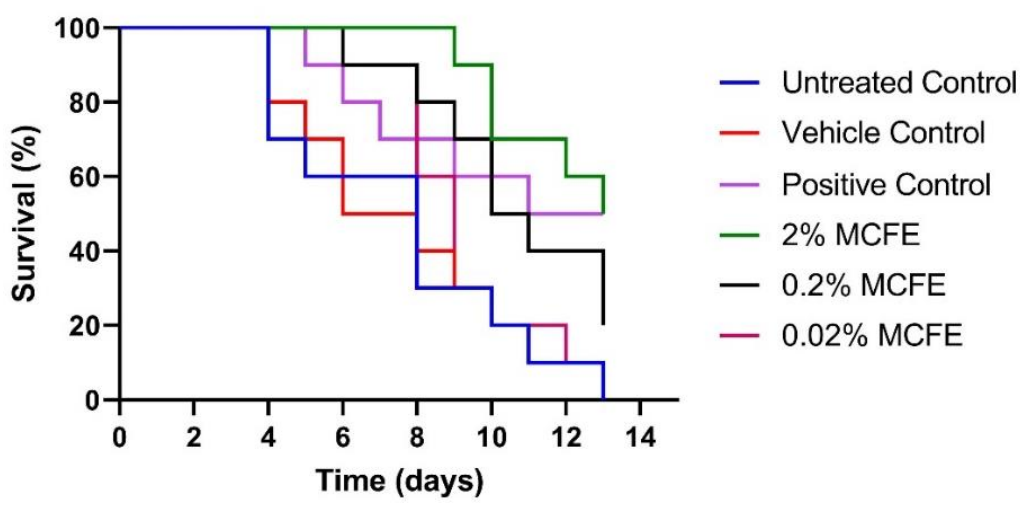

Figure 3. Survival of D. melanogaster after administration of MCFE. Adult fruit flies aged 5-8 days were divided into different groups and subjected to intended treatments. Flies that received no additional treatment were designated as no treatment control, while the ones treated with ethanol $96 \%$ or dexamethasone were assigned as the vehicle control or positive control group, respectively. MCFE, Momordica charantia L. fruit extract 
However, the mechanism of action on how such an anti-inflammatory effect was exerted and whether it has any implication on the survival of organisms experiencing autoinflammatory diseases remains unknown. In this study, we carried out a survival analysis to investigate whether MCFE is beneficial to an in vivo model system experiencing autoinflammatory phenotypical characteristics, the unintended increased production of AMPs. As expected, the administration of MCFE at all concentrations increased the survival of PGRP$\mathrm{LB}^{\Delta}$ D. melanogaster in a manner dependent on the concentration of MCFE (Figure 3). The increased survival of $D$. melanogaster is hypothesized to be a resulting suppressive effect of MCFE on the NF- $\kappa \mathrm{B}-$ mediated immune responses in D. melanogaster.

The longevity of $D$. melanogaster is also related to locomotor activity. Exposure to exogenous compounds or infections can cause locomotor disorders and death [24]. To test the locomotor of MCFE-treated flies, a locomotor test was performed according to the negative geotaxis test protocol [24], with slight modifications.

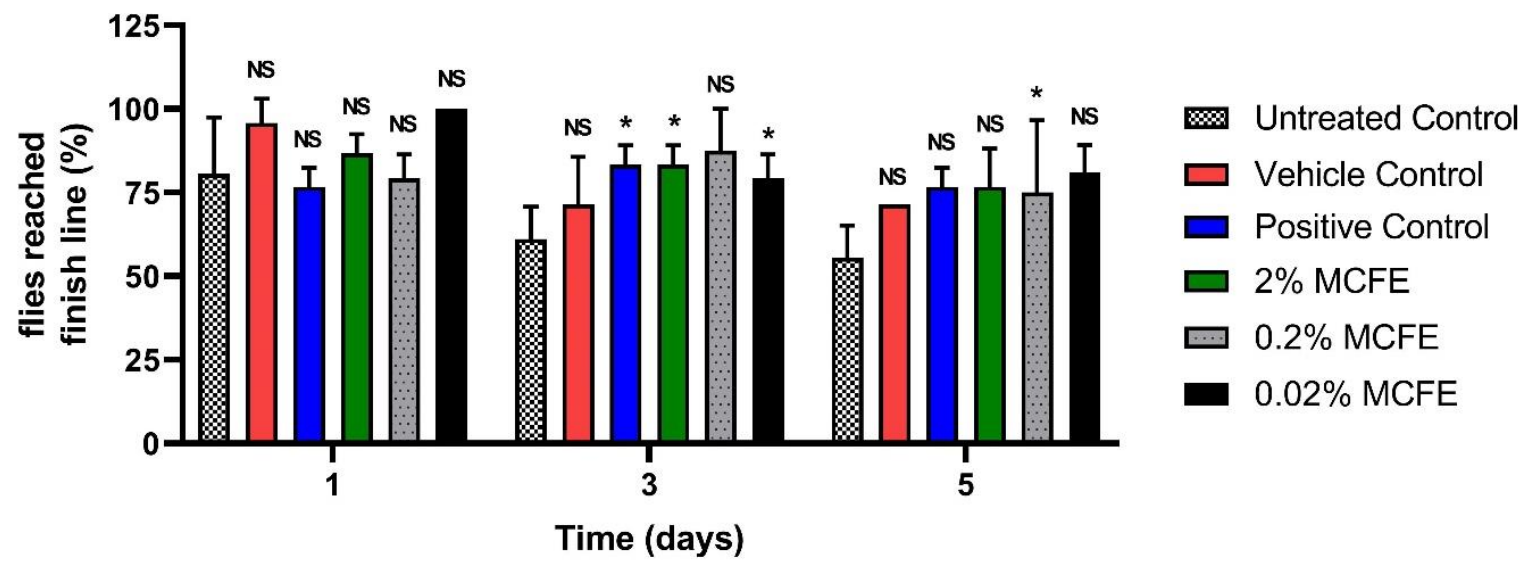

Figure 4. Locomotor activity of $D$. melanogaster after administration of MCFE. Adult fruit flies aged 5-8 days were divided into different groups and subjected to intended treatments. Flies that received no additional treatment were designated as no treatment control, while those treated with ethanol $96 \%$ or dexamethasone were assigned as the vehicle control or positive control group, respectively. MCFE, Momordica charantia L. fruit extract; NS, Non Significant; *, p<0.05.

Based on the results shown in Figure 4, it can be seen that D. melanogaster experienced a slight improvement in the locomotor activity upon MCFE treatment (at 2\% and $0.2 \%$ concentrations) for 3 days but not after. Hence, our results suggested that the lifespan and locomotor activity of $D$. melanogaster are, to some extent, influenced by immune system regulation.

The absence of the PGRP-LB in the PGRP-LB ${ }^{\Delta}$ flies leads to an increase in the NF-kB expression (Figure 2). Previous findings by Kounatidis et al. implicated that increased expression of AMPs downstream of NF- $\mathrm{kB}$ pathway leads to neuronal degeneration, the rapid decline of activity, and shorter lifespan [29]. In line with our findings, downregulation of NF$\kappa \mathrm{B}$ can prolong the lifespan and, to a little extent, improve the locomotor activity of $D$. melanogaster. The administration of MCFE can improve the lifespan and locomotor of D. melanogaster, which may be related to the inhibition of NF-kB activity.

\subsection{Effect of Momordica charantia L. fruit extract on the expression of $N F-\kappa B$-mediated} proinflammatory antimicrobial peptides.

The lifespan of $D$. melanogaster can be influenced by several factors, including the activity of the immune system to overcome bacterial and viral infections [19]. Humans and $D$. melanogaster possess the NF- $\mathrm{BB}$ signaling pathway that plays a tremendous role in activating 
the innate immune system [19]. However, hyperactivation of the immune system, for example, in the condition of cytokine storm, can lead to a dangerous outcome, including death.

Recent reports have suggested that hyperactivation of the innate immune system may lead to cytokine storms $[6,7,12]$. NF- $\kappa \mathrm{B}$ or Relish in $D$. melanogaster is activated by a pathway called the Imd (Immune Deficiency) pathway that expresses numerous target genes, including two antimicrobial peptide genes $d p t$ and $d r o$ [19,30]. Owing to the notion that the shorter lifespan of PGRP-LB ${ }^{\Delta}$ D. melanogaster was possibly due to overexpression of AMPs as a result of NF- $\mathrm{KB}$ hyperactivation, we hypothesized that MCFE might alleviate the expression of AMPs downstream of NF- $\mathrm{BB}$. To test this hypothesis, we analyzed the expression profile of

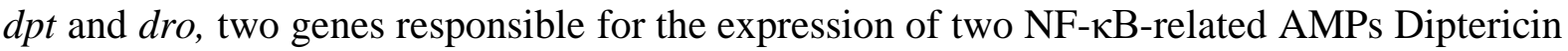
and Drosocin, respectively.

Based on the results shown in Figure 5, the administration of MCFE at all concentrations leads to decreased expression of $d p t$ in $\mathrm{PGRP}_{-\mathrm{LB}^{\Delta}}$ D. melanogaster (Figure 5A). However, a distinct profile was observed in the expression of dro gene (Figure 5B). Apparently, dro expression was downregulated in a concentration-dependent manner; the higher the concentration of MCFE, the lesser the expression of dro in the PGRP-LB ${ }^{\Delta} D$. melanogaster. We believe that such molecular changes lead to the increased survival of PGRP$\mathrm{LB}^{\Delta}$ mutant flies lacking for a negative regulator of NF- $\kappa \mathrm{B}$, as presented in Figure 3.

A

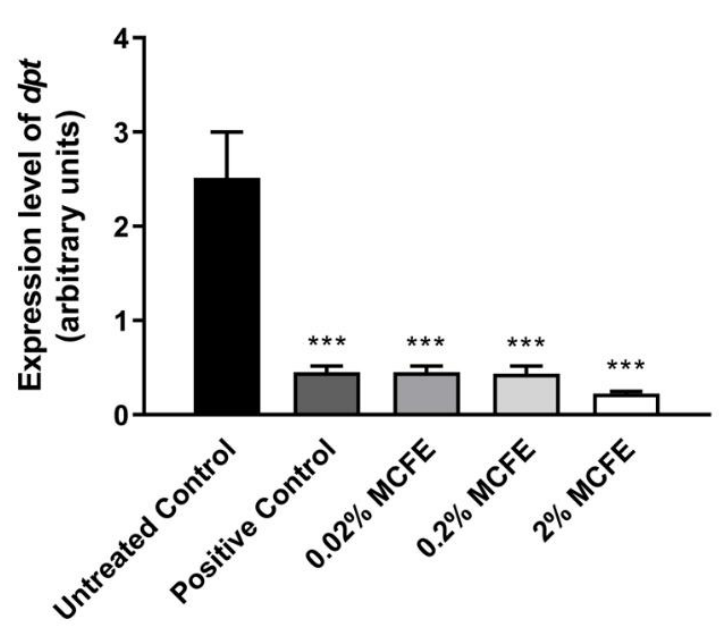

B

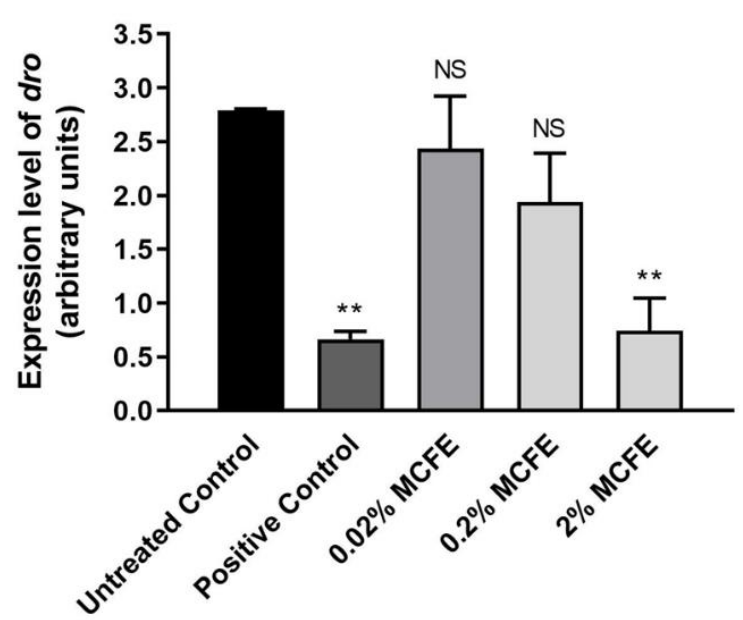

Figure 5. Results of gene expression (A) $d p t$ and (B) $d r o$ in the presence or absence of MCFE treatment. Adult fruit flies aged 5-8 days were divided into different groups and subjected to intended treatments. Flies treated with ethanol $96 \%$ or dexamethasone were assigned as the vehicle control or positive control group, respectively. MCFE, Momordica charantia L. fruit extract; NS, Non Significant; **, $p<0.01 ; * * *, p<0.001$.

In D. melanogaster, activating the Imd pathway leads to the translocation of NF- $\mathrm{BB}$ (Relish) transcription factor into the nucleus [19], triggering the expression of AMP genes, such as $d p t$ and $d r o$, which are homologous to cytokines in humans. Thus, decreased expression of this gene by MCFE suggests the suppression of Relish activation, which in humans may exhibit the same effect as decreasing activation of NF- $\kappa B$. The results obtained in this study indicate that MCFE can suppress the activation of the NF- $\kappa B$ (Imd) pathway. Based on previous studies, suppression of $\mathrm{NF}-\kappa \mathrm{B}$ activation could be a new drug target that may play a specific role in the prevention and/or alleviation of cytokine storms [6,8]. Hence, targeted action of immunosuppressants on the NF- $\kappa \mathrm{B}$ pathway may lead to a better outcome therapy for COVID-19 patients. 


\section{Conclusions}

In the present study, we provided initial experimental in vivo evidence that Momordica charantia L. fruit extract (MCFE) increased the survival of the autoinflammatory disease model of $D$. melanogaster, and such phenotypical changes might be associated with the downregulation of target genes in the NF- $\mathrm{BB}$ pathway. Here, we reported for the first time that MCFE is a potential source of immunosuppressant candidates that works in the NF- $\mathrm{BB}$ (Imd) pathway. Importantly, findings in this study shall warrant forthcoming research in higher model animals or even human subjects.

\section{Funding}

This research received fundings from Student Creativity Program (PKM), Directorate General of Higher Education, Ministry of Education, Culture, Research and Technology, Indonesia, and also partly supported using PDUPT research grant to F.N. (Contract No. 752/UN4.22/PT.02.00/2021) from Directorate General of Higher Education, Ministry of Education, Culture, Research and Technology, Indonesia.

\section{Acknowledgments}

We would like to thank Prof. Yoshinobu Nakanishi and Assoc. Prof. Takayuki Kuraishi (Kanazawa University, Japan) for their generous support in providing the Drosophila melanogaster lines used in this study. We would like to thank Prof. Elly Wahyudin (Biofarmaka laboratory, Hasanuddin University, Indonesia) for her support in providing the research equipment used in this research.

\section{Conflicts of Interest}

We declare that we have no conflict of interest.

\section{References}

1. WHO Coronavirus (COVID-19) Dashboard. Available online: https://covid19.who.int/ (accessed on September 14, 2021).

2. Huang, C.; Wang, Y.; Li, X.; Ren, L.; Zhao, J.; Hu, Y.; Zhang, L.; Fan, G.; Xu, J.; Gu, X.; Cheng, Z.; Yu, T.; Xia, J.; Wei, Y.; Wu, W.; Xie, X.; Yin, W.; Li, H.; Liu, M.; Xiao, Y.; Gao, H.; Guo, L.; Xie, J.; Wang, G.; Jiang, R.; Gao, Z.; Jin, Q.; Wang, J.; Cao, B. Clinical features of patients infected with 2019 novel coronavirus in Wuhan, China. The Lancet 2020, 395, 497-506, https://doi.org/10.1016/S0140-6736(20)30183-5.

3. Zhu, Z.; Lian, X.; Su, X.; Wu, W.; Marraro, G.A.; Zeng, Y. From SARS and MERS to COVID-19: a brief summary and comparison of severe acute respiratory infections caused by three highly pathogenic human coronaviruses. Respiratory Research 2020, 21, 224, https://doi.org/10.1186/s12931-020-01479-w.

4. Nainu, F.; Abidin, R.S.; Bahar, M.A.; Frediansyah, A.; Emran, T.B.; Rabaan, A.A.; Dhama, K.; Harapan, H. SARS-CoV-2 reinfection and implications for vaccine development. Human Vaccines \& Immunotherapeutics 2020, 16, 3061-3073, https://doi.org/10.1080/21645515.2020.1830683.

5. Keam, S.; Megawati, D.; Patel, S.K.; Tiwari, R.; Dhama, K.; Harapan, H. Immunopathology and immunotherapeutic strategies in severe acute respiratory syndrome coronavirus 2 infection. Reviews in Medical Virology 2020, 30, e2123, https://doi.org/10.1002/rmv.2123.

6. Tang, Y.; Liu, J.; Zhang, D.; Xu, Z.; Ji, J.; Wen, C. Cytokine Storm in COVID-19: The Current Evidence and Treatment Strategies. Frontiers in immunology 2020, 11, 1708-1708, https://doi.org/10.3389/fimmu.2020.01708.

7. Birra, D.; Benucci, M.; Landolfi, L.; Merchionda, A.; Loi, G.; Amato, P.; Licata, G.; Quartuccio, L.; Triggiani, M.; Moscato, P. COVID 19: a clue from innate immunity. Immunologic research 2020, 68, 161168, https://doi.org/10.1007/s12026-020-09137-5. 
8. Kircheis, R.; Haasbach, E.; Lueftenegger, D.; Heyken, W.T.; Ocker, M.; Planz, O. NF-кB Pathway as a Potential Target for Treatment of Critical Stage COVID-19 Patients. Frontiers in immunology 2020, 11, https://doi.org/10.3389/fimmu.2020.598444.

9. Shirato, K.; Kizaki, T. SARS-CoV-2 spike protein S1 subunit induces proinflammatory responses via tolllike receptor 4 signaling in murine and human macrophages. Heliyon 2021, 7, e06187-e06187, https://doi.org/10.1016/j.heliyon.2021.e06187.

10. Su, C.-M.; Wang, L.; Yoo, D. Activation of NF-кB and induction of proinflammatory cytokine expressions mediated by ORF7a protein of SARS-CoV-2. Scientific Reports 2021, 11, 13464, https://doi.org/10.1038/s41598-021-92941-2.

11. Nasonov, E.; Samsonov, M. The role of Interleukin 6 inhibitors in therapy of severe COVID-19. Biomed Pharmacother 2020, 131, 110698, https://doi.org/10.1016/j.biopha.2020.110698.

12. Ragab, D.; Salah Eldin, H.; Taeimah, M.; Khattab, R.; Salem, R. The COVID-19 Cytokine Storm; What We Know So Far. Frontiers in immunology 2020, 11, https://doi.org/10.3389/fimmu.2020.01446.

13. Okamoto, H.; Ichikawa, N. The pivotal role of the angiotensin-II-NF-кB axis in the development of COVID19 pathophysiology. Hypertension Research 2021, 44, 126-128, https://doi.org/10.1038/s41440-020-005607.

14. Jia, S.; Shen, M.; Zhang, F.; Xie, J. Recent Advances in Momordica charantia: Functional Components and Biological Activities. International Journal of Molecular Sciences 2017, 18, 2555, https://doi.org/10.3390/ijms18122555.

15. Patel, R.; Mahobia, N.; Upwar, N.; Waseem, N.; Talaviya, H.; Patel, Z. Analgesic and antipyretic activities of Momordica charantia Linn. fruits. Journal of advanced pharmaceutical technology \& research 2010, 1, 415-418, https://doi.org/10.4103/0110-5558.76441.

16. Chao, C.-Y.; Sung, P.-J.; Wang, W.-H.; Kuo, Y.-H. Antiinflammatory effect of Momordica charantia in sepsis mice. Molecules (Basel, Switzerland) 2014, 19, 12777-12788, https://doi.org/10.3390/molecules190812777.

17. Nainu, F.; Nakanishi, Y.; Shiratsuchi, A. Fruit fly as a model organism in the study of human diseases and drug discovery. Journal of Center for Medical Education Sapporo Medical University 2019, 21-32.

18. Pandey, U.B.; Nichols, C.D. Human disease models in Drosophila melanogaster and the role of the fly in therapeutic drug discovery. Pharmacological reviews 2011, 63, 411-436, https://doi.org/10.1124/pr.110.003293.

19. Buchon, N.; Silverman, N.; Cherry, S. Immunity in Drosophila melanogaster--from microbial recognition to whole-organism physiology. Nature reviews. Immunology 2014, 14, 796-810, https://doi.org/10.1038/nri3763.

20. Paredes, Juan C.; Welchman, David P.; Poidevin, M.; Lemaitre, B. Negative Regulation by Amidase PGRPs Shapes the Drosophila Antibacterial Response and Protects the Fly from Innocuous Infection. Immunity 2011, 35, 770-779, https://doi.org/10.1016/j.immuni.2011.09.018.

21. Nainu, F.; Asri, R.M.; Arsyad, A.; Manggau, M.A.; Amir, M.N. In vivo antibacterial activity of green algae Ulva reticulata against Staphylococcus aureus in Drosophila model of infection. Pharmacognosy Journal 2018, 10, 993-997, https://doi.org/10.5530/pj.2018.5.169.

22. Ahsan, M.; Gonsales, A.V.; Sartini, S.; Wahyudin, E.; Nainu, F. In vivo anti-staphylococcal activity of roselle (Hibiscus sabdariffa L.) calyx extract in Drosophila model of infection. Journal of Herbmed Pharmacology 2019, 8, 41-46, https://doi.org/10.15171/jhp.2019.07.

23. Harborne, J.B. Plant Phenolics; Academic Press: 1989; Vol. 1, 1-552, https://www.sciencedirect.com/bookseries/methods-in-plant-biochemistry/vol/1/suppl/C.

24. Linderman, J.A.; Chambers, M.C.; Gupta, A.S.; Schneider, D.S. Infection-related declines in chill coma recovery and negative geotaxis in Drosophila melanogaster. PLOS ONE 2012, 7, e41907, https://doi.org/10.1371/journal.pone.0041907.

25. Nainu, F.; Asri, R.M.; Djide, M.N.; Ahsan, M.; Arfiansyah, R.; Sartini, S.; Alam, G. Protective effect of green algae Ulva reticulata against Pseudomonas aeruginosa in Drosophila infection model. HAYATI Journal of Biosciences 2019, 26, 163-171, https://doi.org/10.4308/hjb.26.4.163.

26. Nainu, F.; Djide, M.N.; Subehan, S.; Sartini, S.; Roska, T.P.; Salim, E.; Kuraishi, T. Protective Signatures of Roselle (Hibiscus sabdariffa L.) Calyx Fractions against Staphylococcus aureus in Drosophila Infection Model. HAYATI Journal of Biosciences 2020, 27, 306-313.

27. Barbosa-Filho, J.M.; Piuvezam, M.R.; Moura, M.D.; Silva, M.S.; Lima, K.V.B.; da-Cunha, E.V.L.; Fechine, I.M.; Takemura, O.S. Anti-inflammatory activity of alkaloids: A twenty-century review. Revista Brasileira de Farmacognosia 2006, 16, 109-139.

28. Maleki, S.J.; Crespo, J.F.; Cabanillas, B. Anti-inflammatory effects of flavonoids. Food Chem 2019, 299 , 125124, https://doi.org/10.1016/j.foodchem.2019.125124.

29. Kounatidis, I.; Chtarbanova, S.; Cao, Y.; Hayne, M.; Jayanth, D.; Ganetzky, B.; Ligoxygakis, P. NF-кB Immunity in the Brain Determines Fly Lifespan in Healthy Aging and Age-Related Neurodegeneration. Cell reports 2017, 19, 836-848, https://doi.org/10.1016/j.celrep.2017.04.007.

30. Neyen, C.; Bretscher, A.J.; Binggeli, O.; Lemaitre, B. Methods to study Drosophila immunity. Methods 2014, 68, 116-128, https://doi.org/10.1016/j.ymeth.2014.02.023. 